\title{
Melamine formaldehyde: curing studies and reaction mechanism
}

\author{
Dyana J Merline, Sulafudin Vukusic and Ahmed A Abdala
}

\begin{abstract}
Melamine formaldehyde (MF) resin was synthesized by the reaction between melamine and formaldehyde under alkaline condition in tetrohydrofuran medium with 1:3 melamine to formaldehyde molar ratio. The synthesized resins were characterized by differential scanning calorimetry (DSC), Fourier transform infrared spectroscopy (FTIR) and thermal gravimetric analysis (TGA). Curing and reaction mechanism was studied by thermal and spectroscopic analysis. Two exothermic peaks were observed in the DSC analysis indicating a two-step crosslinking reaction process was correlated to TGA analysis. FTIR studies at different temperatures explained the two-stage curing mechanism which is concurring with the DSC data. At a temperature range of $140-160^{\circ} \mathrm{C}$, reversible demethylolation is dominating to the crosslinking reaction. At temperature $>160^{\circ} \mathrm{C}$, the crosslinking reaction dominates. On the basis of DSC and FTIR data, a possible crosslinking reaction route was derived and explained. The first stage of curing is the conversion of methylol groups to primary amine and the second stage is the crosslinking of methylol groups to the final product, methylene bridges. The thermal stability of the methylol groups, methylene bridges and the triazine ring, as well as the evaporation of effluents at different stages of curing, are also discussed based on combined TGA and DSC results.
\end{abstract}

Polymer Journal (2013) 45, 413-419; doi:10.1038/pj.2012.162; published online 17 October 2012

Keywords: curing; melamine formaldehyde; thermal properties; thermosetting resin

\section{INTRODUCTION}

Melamine (1, 3, 5-triamino-2, 4, 6-triazine) formaldehyde (MF) is one of the hardest and stiffest thermosetting polymers, which provides good properties and performance. It is an amino resin and has various material advantages, such as transparency, better hardness, thermal stability, excellent boil resistance, scratch resistance, abrasion resistance, flame retardant, moisture resistance and surface smoothness, which lead MF to large industrial applications. ${ }^{1}$ These polymers were originally used as wood adhesives and have now found applications in flooring and decorative laminates, molding compounds, coatings and adhesives. ${ }^{2,3,4} \mathrm{MF}$ resins are incorporated in wide variety of products that are valued for its toughness and relative ease of manufacture. ${ }^{5}$ The curing behavior and the degree of crosslinking of MF resin determine the tailored product properties such as mechanical, thermal and electrical properties. ${ }^{6}$ Cured MF polymers are sufficiently hard and exhibit high resistance against temperature, chemicals and hydrolysis, making them suitable for interior working surfaces. ${ }^{7}$ If the resin is not cured properly, MF will lack mechanical strength and surface finishes. For example, MF impregnated papers will lack hardness, durability, brilliance and resistance to hydrolysis and chemical agents. ${ }^{8}$ The condensation reaction and the resulting structure of MF resins vary significantly with the reaction conditions such as molar ratios of the reactants, $\mathrm{pH}$ and reaction temperature profiles during resin preparation. ${ }^{9,10}$ Thus, curing studies of MF resins finds immense importance.

Several research groups have studied the reaction of melamine with formaldehyde. Studies on the addition reaction between melamine and formaldehyde by means of reversed-phase liquid chromatography has been reported. ${ }^{11}$ All of the nine methylol melamines could be assigned and the technique can also be applied to the quantitative analysis of methylol melamines in the reaction mixtures. Several authors $^{12-14}$ have studied the reactions and structures of soluble MF resins by means of ${ }^{13} \mathrm{C}$ nuclear magnetic resonance (NMR). The structure elucidation of melamine-formaldehyde-polyvinylpyrrolidone by ${ }^{1} \mathrm{H}$ NMR and ${ }^{13} \mathrm{C}$ NMR has been reported. ${ }^{15}$ The methylol, methylene and methylene ether structures were assigned. Several studies on the kinetics of the addition reaction between melamine and formaldehyde in aqueous phase during the initial stage of resin formation have also been explained. ${ }^{11,16,17}$

Cured resins are, because of their insolubility, more difficult to characterize chemically. Cross-polarization magic angle spinning (CP-MAS) ${ }^{13} \mathrm{C}$ NMR, CP-MAS ${ }^{15} \mathrm{~N}$ NMR and Fourier transform infrared spectroscopy (FTIR) spectroscopy have already been utilized for the investigation of the chemical reactions that occur during condensation. Curing studies of MF resins by high-resolution solid state ${ }^{13} \mathrm{C}$ NMR spectra indicate the conversion of free methylo1 
groups to methylene linkages throughout curing. However, the methylene ether links overlap with the residual methylol groups, so it is not clear from these spectra whether or not there are many residual unreacted methylol groups. ${ }^{13,8}$ Information concerning the relative proportions of methylol groups, methylene and methylene ether linkages could be obtained more rapidly from CP-MAS ${ }^{13} \mathrm{C}$ NMR. FTIR spectroscopy has been shown to have only limited capabilities in this regard due to the high number of slightly different structures in the MF resins which result in very broad and overlapping absorption bands. ${ }^{18}$ Thermogravimetry/infrared coupling analysis has also been carried out to determine the effluents during the curing process. ${ }^{19-21} \mathrm{MF}$ polycondensate with high molecular weight and high processing thermostability was prepared with variable melamine: formaldehyde ratios ranging from 1:1.33 to 1:4, which acts as the formaldehyde absorbent by the addition reaction of the hydrogen on the amine groups with the formaldehyde produced by the decomposition of polyoxymethylene under oxygen and heat. ${ }^{22}$ Lower crosslinking degree of MF polycondensate was observed at lower formaldehyde ratio (melamine: formaldehyde ratio of 1: 1.33), which was unstable and decomposed during the thermal weight loss analysis. On the other hand, at very high formaldehyde content, the unreacted hydrogen on the MF molecules was not sufficient to have the role as formaldehyde absorbent of polyoxymethylene.

The MF resin formation consists of two stages: methylolation and condensation. The first attempt to investigate the methylolation and condensation reactions was by Okano and Ogata. ${ }^{9}$ In the first step of methylolation reaction, melamine reacts with formaldehyde producing a series of nine distinct methylol melamine from monohexamethylol melamine. The second step of condensation reaction leads to the formation of large number of different oligomers containing methylene and methylene ether bridges. ${ }^{4,8,10,23,24}$

The ratio of formation of two bridges during condensation reaction depends on the $\mathrm{pH}$ of the reaction medium. If the $\mathrm{pH}$ is relatively low, $7-8$, methylene bridges dominate whereas at high $\mathrm{pH}$ values above 9 , ether bridges are favored. ${ }^{25}$ The cure chemistry and the network formation of two acrylic copolymer resins crosslinked with different MF-based crosslinkers have been studied by Bauer and Dickie ${ }^{26}$ using infrared spectroscopy (IR) spectroscopy, which gives the extent of reaction of the hydroxy and carboxy groups of acrylic copolymer with the methylol group of the MF crosslinker as function of resin composition, melamine type, concentration, cure temperature and cure time. With the data obtained and using a statistical model, effective crosslink density was calculated. The crosslinking chemistry, network formation and degradation of melamine-crosslinked high solids coating have been studied by David. ${ }^{27}$ Author proposed a specific acid-catalyzed mechanism for fully alkylated melamine and a general acid-catalyzed mechanism for partially alkylated melamine. Crosslinking reaction of a highly substituted methylated MF resin with hydroxyl functional polyester analyzed by FTIR has been reported. ${ }^{28}$ Early stage of cure leads to the formation of ether crosslinks before the consumption of all hydroxyl groups and the formation of methylene bridges proceeds through an ether intermediate. FT-Raman spectroscopy in combination with ${ }^{13} \mathrm{C}$ NMR and liquid chromatography has been used for the elucidation of methylolation and ether-methylene-bridge formation in MF resin. ${ }^{29}$ Crosslinking of the polyurethane dispersions with MF resin leads to co-condensation reaction has been studied by Mequanint and Sanderson. ${ }^{30}$

The reaction mechanisms and the pathways involved in the crosslinking of MF resins and the resulting chemical structures are complex. Cured resins are, because of their insolubility, more difficult to characterize chemically. Even though the curing processes of MF resins are well understood on an empirical basis, there is scope for methods that will provide a more detailed understanding of the chemical reactions that occur during condensation. Research work that has been carried out so far mainly explains the elucidation of methylolmelamines and its reaction path. Only few attempts have been done on the crosslinking reaction mechanism. The crosslinking reaction of MF resin in water that leads to the formation of ether bridge has been explained. ${ }^{31}$ The crosslinking mechanism of fully alkylated and partially alkylated MF resin with catalyst has been studied by Blank. ${ }^{32}$ Author found the mechanism by analyzing the volatiles formed during the crosslinking reaction by gas chromatography. Specific acid catalyst was found to be the catalyzing mechanism for fully alkylated MF resin, whereas for the partially alkylated MF resin, the level of formaldehyde content in the reaction volatiles indicated a demethylolation and subsequent catalysis was found to be the crosslinking mechanism. Anderson et al. ${ }^{33}$ studied the initial methylolation and the subsequent thermally induced condensation reaction involves the formation of ether links that readily decomposes into methylene link at above $135^{\circ} \mathrm{C}$ and this reaction sequence is also accompanied by a demethylolation yielding free amine. Still studies are progressing to find a complete reaction mechanism of MF resin. Even though the reversible demethylolation occurring during the cure reaction of MF resin has been cited with the presence of a catalyst and without the presence of a catalyst, still there is no clear idea at what temperature range demethylolation dominates to the crosslinking reaction and also the temperature where crosslinking reaction dominates to demethylolation for a pure non-alkylated MF resin. Even though many research works have been done so far related to the curing studies of MF, we proposed clarity in the reaction mechanism by thermal and spectroscopic tools that simplify the doubts of MF reaction stages, temperatures and the reaction route. Nowhere in the literature explains the two exothermic peaks observed in differential scanning calorimetry (DSC), even though it is known that the reaction mechanism of MF proceeds in two steps cited by several authors. ${ }^{31-33}$ We systematically explains the exact temperature at which the each reaction step is taking place for a non-alkylated MF resin without a catalyst proved by FTIR and consequently a perfect reaction mechanism route has been put forward. The main focus of this paper is to correlate the DSC, spectral and thermal gravimetric analysis (TGA) thermograms of MF resin that are cured thermally for the curing studies. The synthesis and characterization of MF resin are also explained. Characterization techniques such as DSC, FTIR and TGA were used for the studies. Correlating the DSC and TGA thermograms highlights the novelty in the present work.

\section{EXPERIMENTAL PROCEDURE}

\section{Materials}

Melamine (Fluka, Ronkonkoma, NY, USA) and formaldehyde solution 35\% by weight (Sigma Aldrich, Munich, Germany) were used to synthesize the MF resin. Tetrohydrofuran (THF, Fluka) was used as solvent.

\section{MF resin synthesis}

MF resin was prepared by the condensation of melamine and formaldehyde. Aqueous solution of formaldehyde ( $35 \%$ by weigh) was used in this study. Before reaction, $\mathrm{pH}$ value of the formaldehyde solution was maintained to 8.5-9 by using $1 \mathrm{~N} \mathrm{NaOH}$ solution. 1:3 Molar ratio of melamine/formaldehyde was used for the reaction. Eight grams $(0.064 \mathrm{~mol})$ melamine and $15 \mathrm{ml}$ THF were taken in a round-bottomed flask equipped with magnetic stirrer The reaction bath was kept at $65^{\circ} \mathrm{C}$. Fifteen milliliter $(0.192 \mathrm{~mol})$ formaldehyde was added to the above mixture and stirred until melamine is completely dissolved which is recognized by a color change from a white suspension to 
a clear solution. Then the reaction was continued for further $15 \mathrm{~min}$. The clear solution was cool down. Cooling was carried out at room temperature for $30 \mathrm{~min}$. Solvent was removed by rotary evaporator and further by heating at $50{ }^{\circ} \mathrm{C}$ under vacuum overnight. The resulting uncured powder sample was used for further studies.

\section{Preparation of partially and fully cured samples}

To study the structure at different crosslinking stages, three methylolmelamine samples, $1 \mathrm{~g}$ each, were heated in a convection oven from room temperature to 160,170 , and $195^{\circ} \mathrm{C}$ at a heating rate of $5^{\circ} \mathrm{C} \mathrm{min}{ }^{-1}$. Once the corresponding temperatures have been reach each sample has been taken out from the oven, air dried to room temperature, and used for the FTIR study.

\section{Characterization methods}

Differential scanning calorimetry. DSC analysis was carried out in a NETZSCH (Selb, Germany) DSC 204 F1 instrument to study the cure properties of MF resin. Aluminum pans were used as the crucibles for the DSC run. Sample size of between 5-10 mg was used for the analysis. Dynamic scans were made from 50 to $200^{\circ} \mathrm{C}$ at a constant heating rate. Heating rates of $2,5,10,15$ and $20^{\circ} \mathrm{C} \mathrm{min}^{-1}$ were used to study the curing properties of MF resin. For the DSC run, aluminum pans are pierced in order to evaporate any effluents coming out during the scan. All DSC runs were carried out in an inert atmosphere of $\mathrm{N}_{2}$.

Fourier transform infrared spectroscopy. FTIR studies were performed in a Thermo Scientific (Barrington, IL, USA) Nicolet IS10 spectrophotometer. The samples were scanned in the form of $\mathrm{KBr}$ pellets containing $1 \mathrm{mg}$ of the sample and $100 \mathrm{mg}$ of $\mathrm{KBr}$ powder. Spectrum was recorded in the wavenumber range of $4000-500 \mathrm{~cm}^{-1}$ by signal averaging of 32 scans at a resolution of $4 \mathrm{~cm}^{-1}$. The spectra were recorded as percentage transmittance versus wavenumber.

Thermogravimetric analysis. Thermogravimetric analysis was performed in a Netzsch STA 409 thermobalance apparatus. Dynamic temperature scan was performed from 50 to $600{ }^{\circ} \mathrm{C}$ at a constant heating rate of $5^{\circ} \mathrm{C} \mathrm{min}{ }^{-1}$. Analysis was carried out in an inert atmosphere of nitrogen gas with a flow rate of $20 \mathrm{ml} \mathrm{min}^{-1}$. About $15-25 \mathrm{mg}$ of the sample was placed in a ceramic crucible to perform the TGA analysis.

\section{RESULTS AND DISCUSSION}

\section{Polymerization and formulation of MF resin}

Polymerization (curing) of melamine to formaldehyde occurs in two stages. The first step of methylolation is the addition reaction of formaldehyde to melamine that can be carried out in an aqueous phase under neutral or basic conditions or under solvent medium. ${ }^{34}$ The second stage of curing mainly consists of two types of condensation reactions leading to the formation of methylene and methylene ether bridges that can be cured either thermally or under acidic/alkaline conditions. ${ }^{21,23,30}$ During curing, chain extension and crosslinking takes place to form an insoluble and infusible threedimensional network. In this study, stoichiometric ratio of melamine: formaldehyde was chosen as 1:3. The first step of methylolation was carried out in solvent THF. Resulted methylolmelamine was cured thermally at $195^{\circ} \mathrm{C}$ for $45 \mathrm{~min}$.

\section{Curing studies}

FTIR analysis was carried out to identify the first stage and the second stage reaction completion. Figure 1 shows the FTIR spectra of melamine, methylolmelamine and the cured resin. The spectra of methylolmelamine (Figure 1) show absorbances at $3330 \mathrm{~cm}^{-1}$ and $3190 \mathrm{~cm}^{-1}$ correspond to the stretching vibrations of secondary amine. The peak at $2960 \mathrm{~cm}^{-1}$ is due the $\mathrm{C}-\mathrm{H}$ stretching vibration of methylol group. $-\mathrm{C}=\mathrm{N}$ ring vibration is observed at $1530 \mathrm{~cm}^{-1}$. The peak at 1456 and $1388 \mathrm{~cm}^{-1}$ corresponds to the methylene C-H bending vibrations. The absorbance at $1182 \mathrm{~cm}^{-1}$ corresponds to

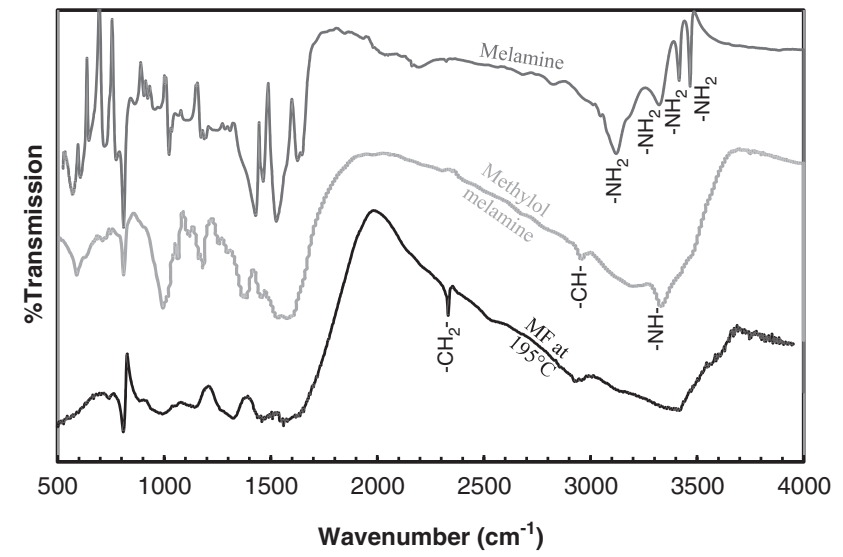

Figure 1 FTIR spectra of melamine, methylolmelamine and cured MF. A full color version of this figure is available at Polymer Journal online.

aliphatic $\mathrm{C}-\mathrm{N}$ vibration. The absorbance at $1110 \mathrm{~cm}^{-1}$ corresponds to $\mathrm{C}-\mathrm{O}$ stretching vibration. The peak at $1060 \mathrm{~cm}^{-1}$ corresponds to the stretching vibration of ether group. The absorptions at 994 and $590 \mathrm{~cm}^{-1}$ correspond to $\mathrm{C}-\mathrm{H}$ out of plane deformations. Bending vibration of triazine ring was found at $810 \mathrm{~cm}^{-1}$. From this spectrum of MF resin, it is understood that the melamine has reacted to methylol groups and there is no residual melamine and formaldehyde remains. Spectra of cured MF (Figure 1) show the $\mathrm{N}-\mathrm{H}$ stretching frequency of secondary amine at $3416 \mathrm{~cm}^{-1}$. Methylene $\mathrm{C}-\mathrm{H}$ bending vibration appeared at $1456 \mathrm{~cm}^{-1}$. And there occurs a generation of new strong peak at $2338 \mathrm{~cm}^{-1}$ that corresponds to the stretching vibration of bridged $\mathrm{CH}_{2}$ group, which gave a strong evidence for methylene bridge formation. ${ }^{15}$ And also there is a decrease in the intensity of the peaks at 1556 and $1437 \mathrm{~cm}^{-1}$ that corresponds to the methylene bending vibrations. This decrease in intensity may possibly due to the extent of crosslinking. From this FTIR interpretation, it was confirmed that the melamine has reacted and crosslinked during the thermal curing process. IR spectral data of the methylolmelamine and cured MF are compiled in Table 1.

To study the curing of MF resins, DSC analysis was carried out by performing dynamic scans were made from 50 to $200^{\circ} \mathrm{C}$ at variable heating rates of $2,5,10,15$ and $20^{\circ} \mathrm{C} \mathrm{min}^{-1}$.

Figure 2 shows the DSC thermograms of an MF resin at different heating rates. The initial increase in the exothermal enthalpy indicates the start of the resin crosslinking. After reaching a maximum, the enthalpy decreases revealing some kind of reaction completion and then again the enthalpy increases and then decreases until the resin crosslinking reaction completes. Hence, the DSC thermograms exhibit two exotherms, indication of two steps of reaction occurring during the thermal curing process. Indication of two-step reaction is further supported by FTIR analysis, which will be discussed later.

Table 2 shows the peak temperatures with variable heating rates for the MF resin. As the heating rate increased, the two peak temperatures of MF resin also increased indicating a slower cure rate of the resin. The evolution of two exothermic peaks implies that the crosslinking of melamine to formaldehyde proceeds in a two-step reaction process. The first exotherm may be attributed to the reversible reaction of methylolmelamine whereas the second exotherm corresponds to the crosslinking of methylol to methylene bridges during the thermal curing process. 
Table 1 IR spectral data of melamine, methylolmelamine and MF resin at variable temperatures

\begin{tabular}{|c|c|c|c|c|c|c|}
\hline Group & Vibration & $\begin{array}{c}\text { Melamine } \\
\left(\mathrm{cm}^{-1}\right)\end{array}$ & $\begin{array}{l}\text { Methylolmelamine } \\
\qquad\left(\mathrm{cm}^{-1}\right)\end{array}$ & $\begin{array}{c}M F \text { at } 162^{\circ} \mathrm{C} \\
\left(\mathrm{cm}^{-1}\right)\end{array}$ & $\begin{array}{c}M F \text { at } 170^{\circ} \mathrm{C} \\
\left(\mathrm{cm}^{-1}\right)\end{array}$ & $\begin{array}{c}M F \text { at } 195^{\circ} \mathrm{C} \\
\left(\mathrm{cm}^{-1}\right)\end{array}$ \\
\hline $\mathrm{N}-\mathrm{H}$ (primary amine) & Stretching & 3467 & & 3464 & & \\
\hline $\mathrm{N}-\mathrm{H}$ (primary amine) & Stretching & 3416 & & 3423 & & \\
\hline $\mathrm{N}-\mathrm{H}$ (primary amine) & Bending & 3321 & & 3337 & & \\
\hline $\mathrm{N}-\mathrm{H}$ (primary amine) & Bending & 3120 & & 3125 & & \\
\hline $\mathrm{N}-\mathrm{H}$ (secondary amine) & Stretching & & 3330 & & 3330 & 3416 \\
\hline $\mathrm{N}-\mathrm{H}$ ( secondary amine) & Stretching & & 3190 & & 3190 & \\
\hline C-H (aliphatic) & Stretching & & 2985 & & 2985 & 2951 \\
\hline $\mathrm{C}-\mathrm{H}$ (bridged & Stretching & & & & & 2338 \\
\hline \multicolumn{7}{|l|}{$\mathrm{CH}_{2}$ group) } \\
\hline $\mathrm{N}-\mathrm{H}$ (primary amine) & Bending & 1625 & & & & \\
\hline $\mathrm{C}=\mathrm{N}$ (ring vibration) & Stretching & 1525 & 1530 & 1530 & 1530 & 1530 \\
\hline C-H (methylene) & Bending & & 1456 & 1437 & 1456 & 1460 \\
\hline $\mathrm{C}-\mathrm{H}$ (methylene) & Bending & & 1388 & 1331 & 1388 & 1323 \\
\hline C-O (aliphatic) & Stretching & & 1160 & & 1160 & 1130 \\
\hline C-O-C (ether group) & Stretching & & 1060 & & 1064 & \\
\hline $\mathrm{C}-\mathrm{H}$ & Bending & 955 & 994 & 992 & 994 & 994 \\
\hline triazine & & 809 & 809 & 816 & 809 & 808 \\
\hline
\end{tabular}

Abbreviation: MF, melamine formaldehyde.

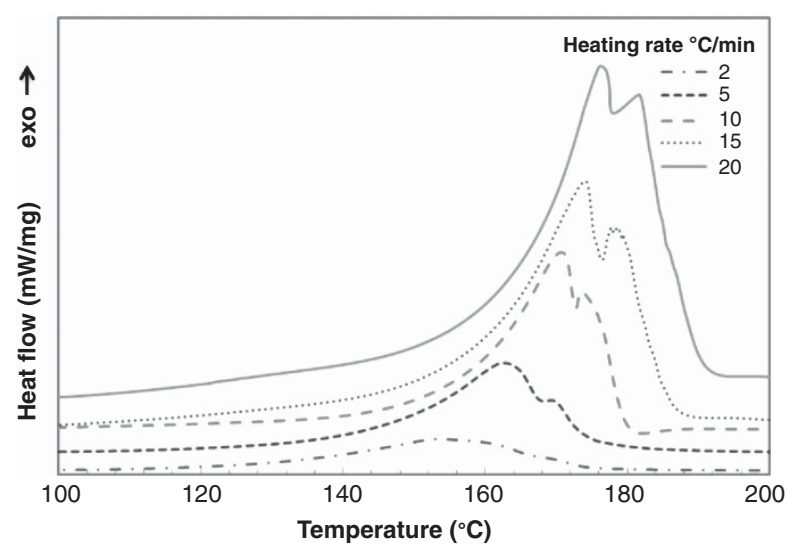

Figure 2 DSC thermograms of MF resin at different heating rates. A full color version of this figure is available at Polymer Journal online.

Table 2 Variation of peak temperatures with heating rates

\begin{tabular}{lcc}
\hline Heating rate $\left({ }^{\circ} \mathrm{C} \mathrm{min}-1\right)$ & Peak $T_{1}\left({ }^{\circ} \mathrm{C}\right)$ & Peak $T_{2}\left({ }^{\circ} \mathrm{C}\right)$ \\
\hline 2 & 153.6 & 166.5 \\
5 & 160.2 & 168.7 \\
10 & 168.5 & 173.4 \\
15 & 174.1 & 177.7 \\
20 & 176.5 & 181.5 \\
\hline
\end{tabular}

To support the DSC investigation for evolution of two exotherms observed, FTIR studies were carried out for the samples cured at two different temperatures $160^{\circ} \mathrm{C}$ and $170{ }^{\circ} \mathrm{C}$, respectively. Figure 3 shows the spectral characterization of MF at two temperatures. Spectral characterization of MF at $160^{\circ} \mathrm{C}$ (Figure 3) shows several distinct peaks. The spectral frequencies between 3464 and $3125 \mathrm{~cm}^{-1}$ corresponds to the primary amine groups of melamine. For comparison, FTIR spectrum of melamine is also shown in Figure 3. Hence, compiling DSC and FTIR studies it suggests that at temperature range

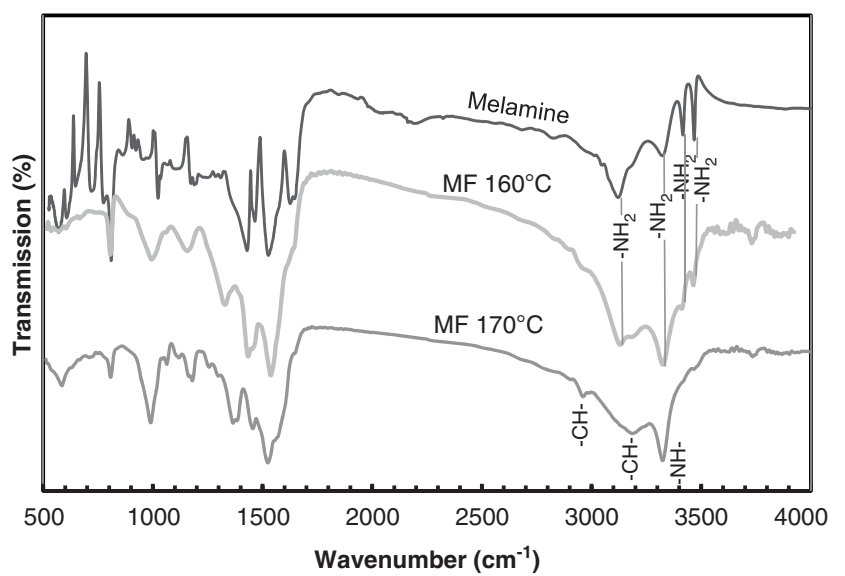

Figure $3 \mathrm{FTIR}$ spectra of melamine, MF resin at $160^{\circ} \mathrm{C}$, and MF resin at $170^{\circ} \mathrm{C}$. A full color version of this figure is available at Polymer Journal online.

from 140 to $160{ }^{\circ} \mathrm{C}$, to some extent the reaction proceeds in a reversible way by converting methylol groups to melamine and thus from the IR data; it is also confirmed that there is no crosslinking reaction taking place at this temperature range, rather, the reverse reaction is taking place. Rest of the absorptions remains the same as methylolmelamine.

Spectra of MF at $170{ }^{\circ} \mathrm{C}$ are shown in Figure 3. It shows the absorbance is the same as methylolmelamine (Figure 1). Spectral data of MF at two different temperatures are also compiled in Table 1. From this IR study at two different temperatures, we attribute the first exotherm observed in DSC to the reverse demethylolation reaction and the second exotherm to the crosslinking reaction of MF resin.

It is evident that the crosslinking mechanism of MF proceeds in two different steps as indicated by DSC analysis. Although reported mechanism for the condensation step for the curing of MF is suggested to involve methylene and ether bridges as intermediate condensation products and these intermediates transform to desired insoluble and infusible MF resins during the final curing process. ${ }^{35}$ However, this mechanism cannot explain the presence of melamine as 
an intermediate product as confirmed by the presence of $-\mathrm{NH}_{2}$ groups in the FTIR spectrum for sample heated from 140 to $160{ }^{\circ} \mathrm{C}$ and disappearance of these groups from the spectrum of the samples heated above $160^{\circ} \mathrm{C}$. Therefore, based on the DSC and the FTIR results, which are in agreement, we propose a modified mechanism as discussed in the following below.

Methylolation step. This step is well studied and involves direct reaction between melamine and formaldehyde to form methylolamine as follows.

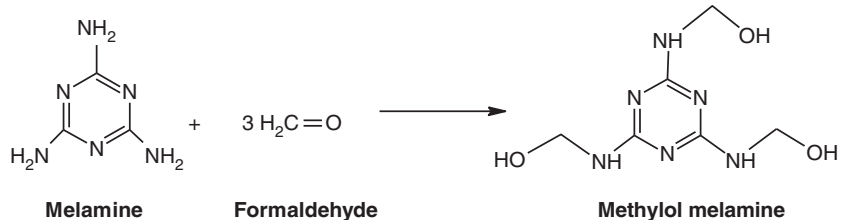

Condensation step. This step is more complex and involves multiple reactions that lead to intermediate ether-linkage-containing compounds. These ether linkages are transformed into methylene linkages by the elimination of formaldehyde as described by Scheme 1 . However, we suggest the existence of another reaction that leads to the formation of the methylene bridges in the final cured product through reaction between methylolamine formed in the methylolation reaction and melamine.
As discussed in the previous sections, melamine is formed by the decomposition of methylolamine at a temperature above $140{ }^{\circ} \mathrm{C}$ as shown in Scheme 2.

This reaction step is confirmed by the presence of the $-\mathrm{NH}_{2}$ groups in the FTIR spectrum for the partially cured sample $\left(\mathrm{T}<160^{\circ} \mathrm{C}\right)$.

Correlating DSC and TGA analysis. Figure 4 shows the TGA and the DSC thermograms of MF resin in the temperature range from 50 to $600{ }^{\circ} \mathrm{C}$. We can define four temperature ranges where the weight losses appeared, from 100 to 180,180 to 350,350 to 390 and 390 to $600{ }^{\circ} \mathrm{C}$, respectively. Correspondingly two exotherms were observed in the DSC thermograms. The first exotherm in the temperature region $100-180^{\circ} \mathrm{C}$ corresponds to the curing reaction of MF resin splits into two, as in the DSC study, which gave support for the cure reaction, and also the weight loss is related to the thermal curing process.

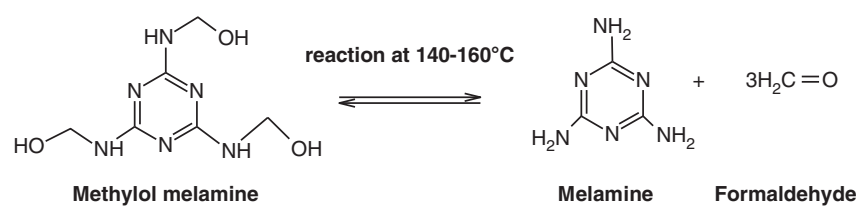

Scheme 2 Formation of melamine by decomposition of methylolamine.<smiles>CCCCOCNc1nc(NCOCNc2nc(NC)nc(NCOCNc3nc(NC)nc(NC)n3)n2)nc(NCOCNc2nc(NCO)nc(NCOCNc3nc(NC)nc(NCO)n3)n2)n1</smiles>

Scheme 1 Reaction mechanism of thermal curing of MF resin. 


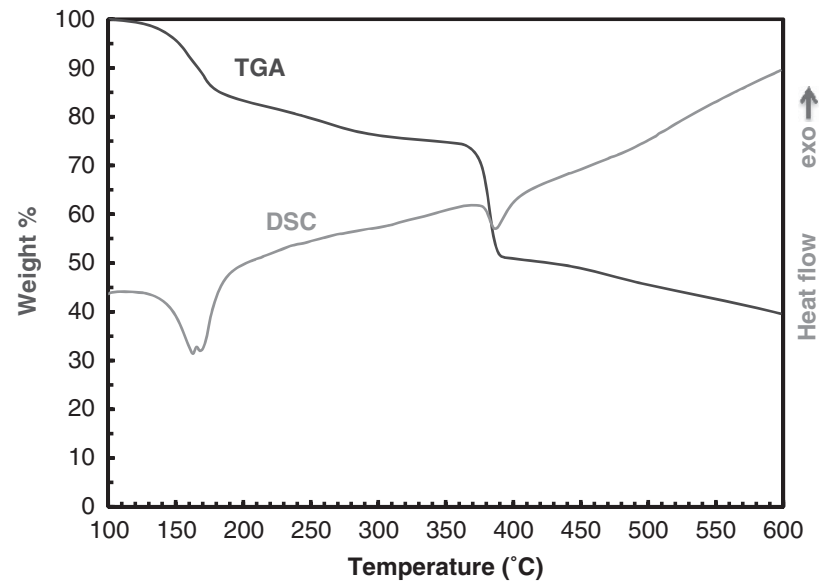

Figure $4 \mathrm{TGA}$ and DSC thermograms of MF resin. A full color version of this figure is available at Polymer Journal online.

Corresponding $12.36 \%$ weight loss is due to the evaporation of water during the condensation process. Of total $12.36 \%$ weight loss, $7.1 \%$ weight loss corresponds to water elimination due to the selfcondensation of methylol groups, leading to ether bridge formation as per the reaction occurring in $140-160{ }^{\circ} \mathrm{C}$ temperature range, and the rest of $5.26 \%$ weight loss is also due to water elimination during the condensation reaction between melamine and the methylol group, which leads to the methylene bridge as per the curing reaction occurring at temperature $>160^{\circ} \mathrm{C}$, as shown in Scheme 1 . The weight $\%$ was calculated based on the 1:3 melamine: formaldehyde mole ratios.

Weight loss of $12.8 \%$ occurs in the temperature range of 180 $350{ }^{\circ} \mathrm{C}$ corresponds to the elimination of formaldehyde from the ether bridge forming methylene bridge as shown below:

$$
\mathrm{M}-\mathrm{NH}-\mathrm{O}_{-}-\mathrm{NH}-\mathrm{M} \stackrel{-\mathrm{CH}_{2} \mathrm{O}}{\longrightarrow} \mathrm{M}-\mathrm{NH} \overbrace{\mathrm{NH}-\mathrm{M}}^{\longrightarrow}
$$

Third, weight loss of $22.8 \%$ observed in the temperature range of $350-390^{\circ} \mathrm{C}$ is attributed to the breakdown of methyelene bridges. ${ }^{20}$ Correspondingly, DSC thermogram shows an exothermic peak. Possible reaction mechanism of the breakage of methylene bridge is shown below:
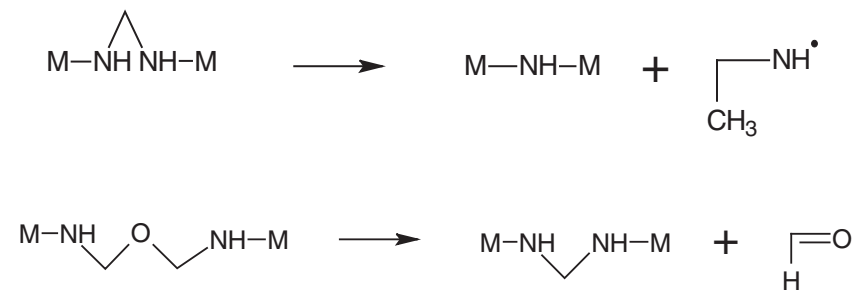

$\mathrm{CH}_{2} \mathrm{NH}$ fragment dimerizes to azomethane, which will dimerize to ethane as follows:

$$
\mathrm{H}_{3} \mathrm{C}-\mathrm{N}=\mathrm{N}-\mathrm{CH}_{3} \longrightarrow 2 \mathrm{CH}_{3}+\mathrm{N}_{2}
$$

Weight loss beyond $390^{\circ} \mathrm{C}$ is attributed to the thermal degradation of the triazine ring, and it is assumed that MF resin progressively deaminates to form $\mathrm{HCN}{ }^{19}$

\section{CONCLUSIONS}

The curing mechanism of MF resin was explained by both thermal and spectroscopic studies. DSC thermogram exhibited two exotherms which gave evidence of two-stage curing process. Subsequent FTIR studies at two exothermic peak temperatures observed in DSC reveal the two-stage crosslinking reaction mechanism. The first step of thermal curing at temperature range of $140-160{ }^{\circ} \mathrm{C}$ is the reverse reaction of methylol groups to melamine and second step occurring at temperature $>160^{\circ} \mathrm{C}$ is the crosslinking reaction. Hence, by the mutual agreement of DSC and FTIR studies, a possible reaction route was derived for the thermal curing process. The TGA characterization clearly shows four successive mass losses in the temperature range of $50-600{ }^{\circ} \mathrm{C}$, which is correlated to the DSC thermogram. Correlation of the DSC and TGA clearly explains the weight loss during the curing process due to the evaporation of water and formaldehyde. As in the DSC study, DSC-TGA thermogram supports the two-step process of the cure reaction by exhibiting two peaks in the same temperature region. First peak at $160^{\circ} \mathrm{C}$ corresponds to the weight loss due to the elimination of formaldehyde due to the reverse reaction of MF resin, and the second peak corresponds to water elimination due to the condensation reaction. Methylene bridges are stable up to $350^{\circ} \mathrm{C}$, and the thermal degradation of triazine ring begins at $400{ }^{\circ} \mathrm{C}$.

1 Bajia, S., Sharma, R. \& Bajia, B. Solid-state microwave synthesis of melamine formaldehyde resin. J. Chem. 6, 120-124 (2009).

2 Park, B. -D. \& Jeong, H. -W. Cure kinetics of melamine-formaldehyde resin/clay/ cellulose nanocomposites. J. Ind. Eng. Chem. 16, 375-379 (2010).

3 Dipak, K. R., Amit, J. P. \& Bhavi, N. N. A Study on composites from casein modified melamine formaldehyde resin. Poly. Plast. Tech. Eng. 45, 293-299 (2006).

4 Anil, K. \& Vimal, K. Modelling and experimental investigation of melamine formalde hyde polymerization. Macromolecules 23, 3729-3736 (1990).

5 El-Sayed, W. S., El-Baz, A. F. \& Othman, A. M. Biodegradation of melamine formaldehyde by micrococcus sp. strain MF-1 isolated from aminoplastic waste water effluent. Int. Biodeteriorat. Biodegradat. 57, 75-81 (2006).

6 Wohnsiedler, H. P. Polymerisation in melamine-formaldehyde molded resins. Ind. Eng Chem. 45, 2307-2311 (1953).

7 Kandelbauer, A., Wuzella, G., Mahendran, A., Taudes, I. \& Widsten, P. Using isoconventional kinetic analysis of liquid melamine-formaldehyde resin curing to predict laminate surface properties. J. Appl. Polym. Sci. 113, 2649-2660 (2009).

8 Kandelbauer, A., Wuzella, G., Mahendran, A., Taudes, I. \& Widsten, P. Model-free kinetic analysis of melamine-formaldehyde resin cure. Chem. Eng. J. 152, 556-565 (2009).

9 Okano, B. M. \& Ogata, Y. Kinetics of condensation of melamine with formaldehyde. J. Am. Chem. Soc. 74, 5728-5732 (1952).

10 Mijatowic, J., Binder, W. H., Kubel, F. \& Kantner, W. Studies on the stability of MF resin solutions: investigations on network formation. Macromol. Symp. 181, 373-382 (2002).

11 Tomita, B. Melamine-formaldehyde resins: molecular species distributions of methylolmelamines and some kinetics of methylolation. J. Poly. Sci. Poly. Chem. Edi. 15, 2347-2365 (1977).

12 Tomita, B. \& Ono, H. Melamine-Formaldehyde resins: constitutional characterization by $\mathrm{C}-13$ fourier transform NMR spectroscopy. J. Poly. Sci. Poly. Chem. Edi. 17 3205-3215 (1979)

13 Ebdon, J. R., Hunt, B. J., O'Rourke, W. T. S. \& Parkin, J. Characterisation of some melamine-formaldehyde condensates and some cured resins by ${ }^{1} \mathrm{H},{ }^{13} \mathrm{C}$ and ${ }^{15} \mathrm{~N} \mathrm{n} . \mathrm{m} . \mathrm{r}$. Spectrosc. Br. Poly. J. 20, 327-334 (1988).

14 Dawbarn, M., Ebdon, J. R. \& Hewitt, S. J. Examination of some melamine formaldehyde adduct by C13 nuclear magnetic resonance. Polymer 19, 1309-1312 (1978).

15 Sing, M. \& Kumar, M. Preparation and characterisation of melamine-formaldehyde polyvinylpyrrolidone polymer resin for better industrial uses over melamine resin. J. Appl. Poly. Sci. 114, 1870-1878 (2009).

16 Gordon, M., Halliwell, A. \& Wilson, T. The Chemistry of Polymerization Processes, SCI Monograph No. 20 (Society of Chemical Industry, London, 1965).

17 Sato, K. \& Naito, T. Studies on melamine resin: kinetics of acid-catalysed condensation of di and trimethylolmelamine. Poly. J. 5, 144-157 (1973).

18 Braun, D. \& Legradic, V. Investigation of the melamine formadehyde reaction products. Ange. Makro. Chemie. 36, 41-55 (1974).

19 Devallencourt, C., Saiter, J. M., Fafet, A. \& Ubrich, E. Thermogravimetry/fourier transform infrared coupling investigations to study the thermal stability of melamine formaldehyde resin. Thermo. Acta 259, 143-151 (1995).

20 Manley, T. R. \& Higgs, D. A. Thermal stability of melamine formaldehyde resins. J. Poly. Sci. Symp. 42, 1377-1382 (1973).

21 Moore, W. R. \& Donnelly, E. Thermal degradation of melamine formaldehyde resins. J. Appl. Chem. 13, 537-543 (1963).

22 Youliang, H., Lin, Y. \& Xiaowen, Z. Synthesis of the melamine-formaldehyde polycondensate and its thermal stabilization effect on polyoxymethylene. Polymer 47, 2649-2659 (2006). 
23 Vo, D. T., Himmelsbach, M., Haunschmidt, M., Buchberger, W., Schwarzinger, C. \& Klambfl, C. W. Improved analysis of melamine-formaldehyde resins by capillary zone electrophoresis-mass spectrometry using ion-trap and quadrupole-time-of-flight mass spectrometers. J. Chromat. A 1213, 83-87 (2008).

24 Berge, A. \& Mejdell, T. Melamine formaldehyde compounds. The active species in acid catalyzed reactions. Polymer 47, 3249-3256 (2006).

25 Naske, R., Dietrich, K., Reinisch, G. \& Refler, G. The initial stage of the reaction of melamine with formaldehyde. J. Macromol. Sci. Chem. A 23, 579-596 (1986).

26 Bauer, D. R. \& Dickie, R. A. Crosslinking chemistry and network structure in organic coatings. I. Cure of melamine formaldehyde/acrylic copolymer films. J. Poly. Sci. Poly. Phys. Ed. 18, 1997-2014 (1980)

27 David, B. R. Melamine/formaldehyde crosslinkers: characterization, network formation and crosslink degradation. Prog. Org. Coat. 14, 193-218 (1986).

28 Wilson, R. C. \& Pfohl, W. F. Study of cross-linking reactions of melamine/formaldehyde resin with hydroxyl functional polyester by generalized 2-D infrared spectroscopy. Vibrat. Spect. 23, 13-22 (2000).
29 Scheepers, M. L., Gelan, J. M., Carleer, R. A., Adriaensens, P. J. \& Vanderzande, D. J. Investigation of melamine-formaldehyde cure by fourier transform Raman spectroscopy. Vibrat. Spect. 6, 55-69 (1993).

30 Mequanint, K. \& Sanderson, R. Nano-structure phosphorus-containing polyurethane dispersions: synthesis and crosslinking with melamine formaldehyde resin. Polymer 44, 2631-2639 (2003).

31 Manfred, R., Hartmut, B., Sergey, I., Valery, P., Paul, L. \& Primachenko, O. N. The reaction mechanism of the transetherification and crosslinking of melamine resins. Macromol. Symp. 217, 431-443 (2004).

32 Blank, W. J. Reaction mechanism of amino resin. J. Coat. Techn. 51, 61-70 (1979). 33 Anderson, I. H., Cawley, M. \& Steedman, W. Melamine-formaldehyde resins: an examination of some model compound systems. Br. Polym. J. 1, 24-28 (1969).

34 Dawbarn, M., Ebdon, J. R. \& Hewitt, S. J. Examination of some melamine formaldehyde adduct by C13 nuclear magnetic resonance. Polymer 19, 1309-1312 (1978).

35 Pizzi, A. Handbook of Adhesive Technology. 2nd edn (eds Pizzi and Mittal) Ch. 32, 653-680 (Marcel Dekker, France, Inc., 2003). 